\title{
Soft X-ray emission lines in the afterglow spectrum of GRB 011211: A detailed XMM-Newton analysis
}

\author{
J. N. Reeves ${ }^{1,2,3}$, D. Watson ${ }^{1}$, J. P. Osborne ${ }^{1}$, K. A. Pounds ${ }^{1}$, and P. T. O’Brien ${ }^{1}$ \\ 1 X-ray and Observational Astronomy Group, Department of Physics and Astronomy, University of Leicester, University Road, \\ Leicester LE1 7RH, UK \\ 2 Laboratory for High Energy Astrophysics, Code 662.0, NASA Goddard Space Flight Center, Greenbelt Road, Greenbelt, \\ MD 20771, USA \\ 3 Universities Space Research Association
}

Received 28 June 2002 / Accepted 6 February 2003

\begin{abstract}
We report on an XMM-Newton observation of the X-ray afterglow of the Gamma Ray Burst GRB 011211, originally detected by Beppo-SAX on 11th December 2001. The early afterglow spectrum obtained by XMM-Newton, observed 11 hours after the initial burst, appeared to reveal decaying $\mathrm{H}$-like $\mathrm{K} \alpha$ emission lines of $\mathrm{Mg}, \mathrm{Si}, \mathrm{S}, \mathrm{Ar}$ and $\mathrm{Ca}$, arising in enriched material with an outflow velocity of order 0.1c (Reeves et al. 2002). This was attributed to matter ejected from a massive stellar progenitor occurring shortly before the burst itself. Here, we present a detailed re-analysis of the XMM-Newton EPIC observations of GRB 011211. In particular, we show that the detection of the soft X-ray line emission appears robust, regardless of detector background, calibration, spectral binning, or the spectral model that is assumed. We demonstrate that thermal emission, from an optically thin plasma, is the most plausible model that can account for the soft X-ray emission, which appears to be the case for at least two burst afterglow spectra observed by XMM-Newton. The X-ray spectrum of GRB 011211 appears to evolve with time after the first $10 \mathrm{ks}$ of the XMM-Newton observation as the Si and $\mathrm{S}$ emission lines are only detected during the first $10 \mathrm{ks}$ of observation. The observations suggest that thermal emission is present during the early afterglow spectrum, whilst a power-law component dominates the latter stages. Finally we estimate the mass of the ejected material in GRB 011211 to be of the order 4-20 solar masses.
\end{abstract}

Key words. gamma rays: bursts - supernovae: general - X-rays: general

\section{Introduction}

The study of X-ray afterglow emission is crucial to understanding the nature of gamma ray bursts and their progenitors, as $\mathrm{X}$-ray spectroscopy can reveal details of the environment of the burst explosion. For instance, observations of some X-ray afterglows with Beppo-SAX, ASCA, and Chandra have revealed strong iron $\mathrm{K} \alpha$ emission lines (e.g. GRB 000214, Antonelli et al. 2000; GRB 991216, Piro et al. 2000; GRB 970828, Yoshida et al. 2001; GRB 970508, Piro et al. 1998), with line equivalent widths of up to several $\mathrm{keV}$; typically the lines are detected at $\sim 3 \sigma$ confidence. An iron K-shell absorption feature was also reported in the prompt Beppo-SAX spectrum of one burst, GRB 990705 (Amati et al. 2000; Lazzati et al. 2001). The line observations appear to support models where the burst explodes into an enriched, high density medium, favoring a massive stellar progenitor for long duration bursts. In many cases (e.g. Piro et al. 2000; Antonelli et al. 2000), large masses of iron are required to account for the line emission. One possible source of the iron is in a shell of distant $\left(R \sim 10^{16} \mathrm{~cm}\right)$

Send offprint requests to: D. Watson, e-mail: wat@star.le.ac.uk material ejected from a supernova (the "supranova" model, Vietri \& Stella 1999) which would have to occur at least several months prior to the burst event, in order to allow sufficient time for large quantities of iron to form (e.g. Vietri \& Stella 1999). Alternatively, the iron K emission could arise through the interaction of a magnetically driven wind with the envelope of the massive progenitor star (Rees \& Meszaros 2000); here the distances involved are much smaller $\left(R \sim 10^{13} \mathrm{~cm}\right)$ and consequently the high masses of iron and long time delays between supernova and burst are not required.

The high throughput of XMM-Newton, compared with Beppo-SAX or Chandra, makes it the best available telescope with which to constrain X-ray line emission in GRBs, potentially providing a powerful diagnostic for discriminating between models of the burst progenitor. In an analysis of the XMM-Newton observation of the afterglow of gamma ray burst GRB 011211, Reeves et al. (2002) reported the first detection of emission lines other than iron in a GRB afterglow spectrum; specifically the decaying line emission from the hydrogenic states of $\mathrm{Mg}, \mathrm{Si}, \mathrm{S}, \mathrm{Ar}$ and $\mathrm{Ca}$. Furthermore the energies of the X-ray lines appeared to be offset from the known redshift for the host galaxy of GRB $011211(z=2.140 \pm 0.001$, 
Holland et al. 2002), implying the line emitting matter was outflowing with a velocity of $\sim 0.1 \mathrm{c}$ from the site of the GRB progenitor. This result was interpreted as possible evidence of a supernova explosion occurring within days of the burst itself, with the X-ray line emission arising from matter in the expanding supernova shell.

Recently, Borozdin \& Trudolyubov (2003) have claimed that the detection of soft X-ray emission lines from light metals in GRB 011211 may not be robust, as the observations could be contaminated by the background of the XMM-Newton EPIC-pn detector. In addition, Rutledge \& Sako (2003) perform simulations which suggest that the significance of the emission features may be lower, at the level (at best) of $\sim 98.8 \%$ confidence. In this paper, we describe our analysis of the XMM-Newton data on GRB 011211 in detail. We confirm that the soft X-ray emission features in GRB 011211 are robust, the set of lines being detected to a good level of confidence (at $>99 \%$ significance in all our tests). Both the F-test and Monte-Carlo simulations are used to determine the statistical significance of the line emission features, making no prior assumption about the rest energies or redshift of the line emitting material. We also demonstrate that the results do not depend on the background subtraction of the EPIC-pn detector, instrument calibration, or the spectral binning used and show that the result is independent of the spectral model that is assumed. In Sect. 2 we review the properties of the burst, the XMM-Newton observations and basic data analysis and in Sect. 3 detail the spectral analysis of the afterglow during the first $5 \mathrm{ks}$ when the lines appear most prominent. The time dependent spectral properties of GRB 011211 are also outlined, whilst a likely mechanism of the line emission is discussed and a mass for the ejected material in GRB 011211 is derived. Throughout this paper we adopt a cosmology of $H_{0}=75 \mathrm{~km} \mathrm{~s}^{-1} \mathrm{Mpc}^{-1}$ and $q_{0}=0.1$. Unless otherwise stated, all errors are quoted at $68 \%$ confidence (i.e. $\Delta \chi^{2}=1.0$ or 2.3 for 1 or 2 interesting parameters respectively).

\section{XMM-Newton observations of GRB 011211}

The Gamma Ray Burst GRB 011211 was first detected on December 11th 2001 at 19:09:21 (UT), by Beppo-SAX (Frontera et al. 2001). The burst duration was $270 \mathrm{~s}$, making GRB 011211 the longest burst yet observed by BeppoSAX, with a peak flux $(40-700 \mathrm{keV})$ of $5 \times 10^{-8} \mathrm{erg} \mathrm{cm}^{-2} \mathrm{~s}^{-1}$. Spectroscopy of the transient optical afterglow revealed several absorption lines at a redshift of $z=2.140 \pm 0.001$ (Holland et al. 2002). Optical imaging (Burud et al. 2001; Fox et al. 2002) has also linked the optical transient to extended emission, the probable host galaxy of magnitude $m_{v}=25.0 \pm 0.5$. Assuming the absorption system arises from the host galaxy of the GRB, then the total equivalent isotropic luminosity for GRB 011211 is $5 \times 10^{52} \mathrm{erg}$.

The XMM-Newton (Jansen et al. 2001) observations of GRB 011211 started at 06:16:56 (UT) on December 12th 2001, $\sim 11$ hours after the initial burst (Santos-Lleo et al. 2001). Data from EPIC (European Photon Imaging Cameras) have been analysed, using both the MOS CCDs (Turner et al. 2001) with the medium filter and pn CCDs (Struder et al. 2001) using the thin filter. All EPIC observations were taken in

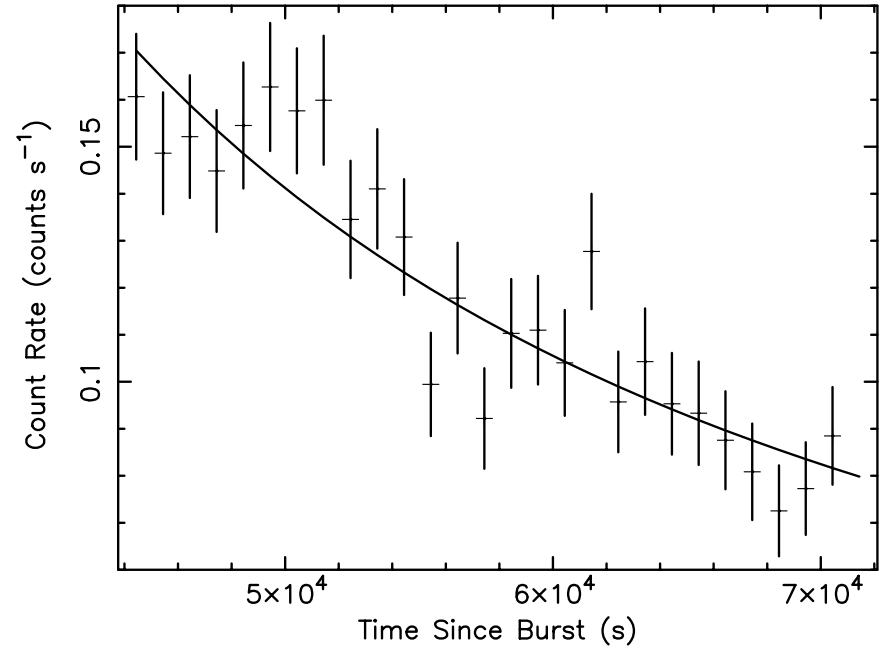

Fig. 1. EPIC $0.2-10 \mathrm{keV}$ lightcurve (coadded pn and MOS). A powerlaw decay fits the data well, the best-fit power-law, (with a decay index of $1.6 \pm 0.1$ ) is plotted. Time since the GRB is plotted on the abscissa.

Full Window mode. The exposure start times were 06:46:59, 07:04:45 and 07:13:30 for MOS-1, MOS-2 and EPIC-pn respectively. Hereafter in the paper we refer to the start-time of observation according to the beginning of the EPIC-pn exposure. The observation end times for EPIC were 15:32:21, 15:32:22 and 15:29:56, for MOS-1, MOS-2 and pn. The total observation duration (for EPIC-pn) was therefore approximately $30 \mathrm{ks}$.

At $4.2 \mathrm{ks}$ into the observation (for $\sim 500 \mathrm{~s}$ ), the spacecraft pointing was moved from its original position at co-ordinates (J2000) $11 \mathrm{~h} 15^{\prime} 16.4^{\prime \prime},-21^{\circ} 55^{\prime} 44.8^{\prime \prime}$, to new boresight coordinates at $11 \mathrm{~h} 15^{\prime} 18.0^{\prime \prime},-21^{\circ} 56^{\prime} 53.0^{\prime \prime}$, in order to place the source further away from the EPIC-pn chip gaps. The timeaveraged $0.2-10 \mathrm{keV}$ flux was $1.7 \times 10^{-13} \mathrm{erg} \mathrm{cm}^{-2} \mathrm{~s}^{-1}$ (corresponding to an $0.6-30 \mathrm{keV}$, rest frame afterglow luminosity of $7 \times 10^{45} \mathrm{erg} \mathrm{s}^{-1}$ ), decreasing during the observation according to a power-law, with decay rate $F(t) \propto t^{-\alpha}, \alpha=1.6 \pm 0.1$ (Fig. 1). The X-ray decay is notably steeper than reported for the same period in the optical ( $\alpha=0.83 \pm 0.04$, Holland et al. 2002).

\subsection{Extraction of the XMM-Newton data}

We initially concentrate our analysis on the first $5 \mathrm{ks}$ of observation, where the prominent soft X-ray emission lines are observed. The analysis focuses on the EPIC-pn spectrum, which due to the use of the thin filter and its higher effective area, has a much higher count rate than the EPIC-MOS (at $0.6 \mathrm{keV}$, the PN count rate is $5.7 \times$ higher than a single MOS detector), having first checked that the MOS and pn data are self-consistent. All data reduction was performed using the XMM-SAS (v5.3).

Figure 2a shows the unscreened EPIC-pn field around GRB 011211, the afterglow itself being the bright source located close to the edge of the EPIC-pn chip gap, but near to the centre of the image. The background count rate is clearly higher around the edges of the pn chip in the unscreened dataset, as noted by Borozdin \& Trudolyubov (2003), who claim that 


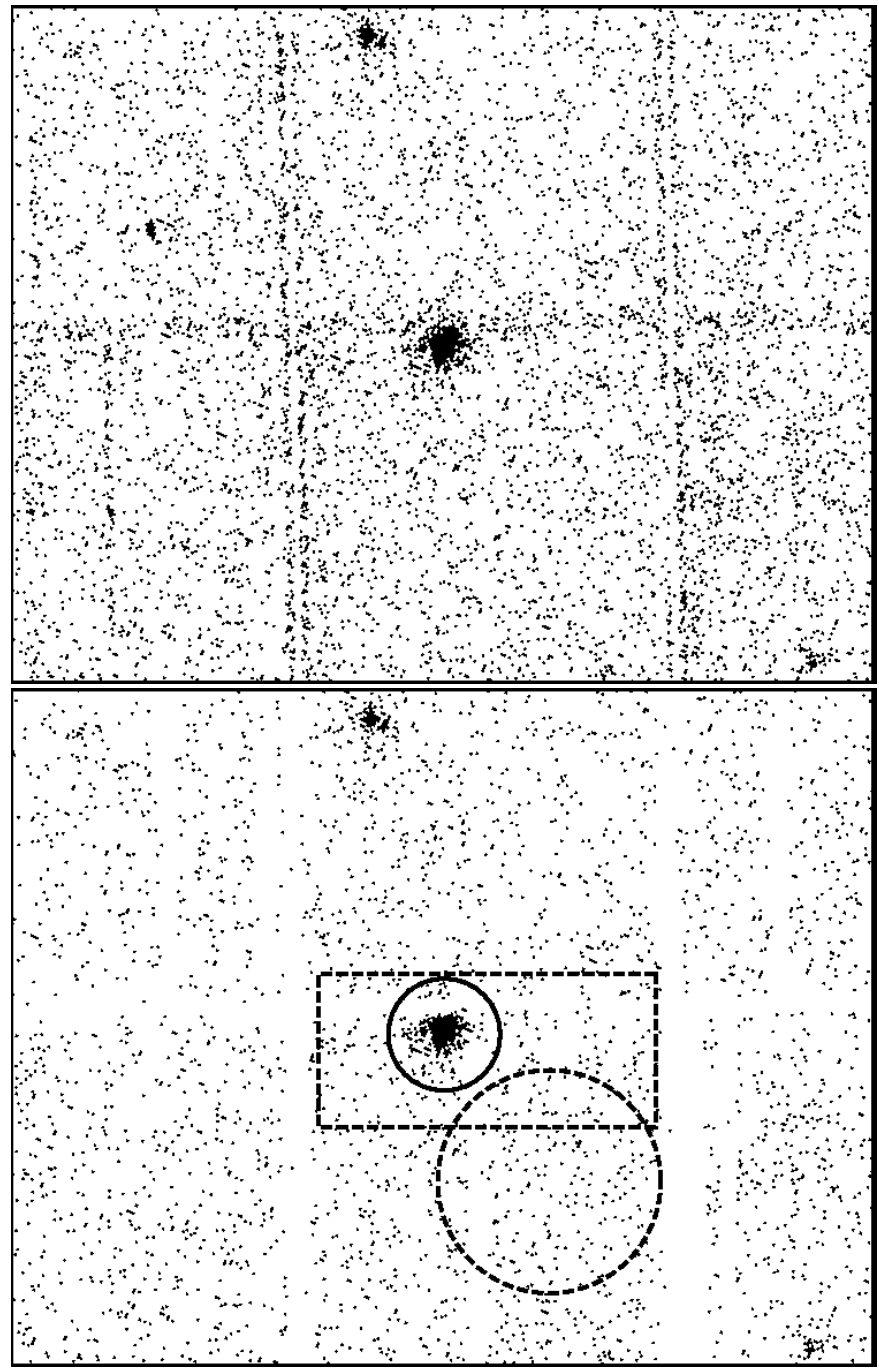

Fig. 2. XMM-Newton images of the field around GRB 011211, during the first $5 \mathrm{ks}$ of observation; a) top shows the unscreened image, whilst b) bottom shows the screened image, used for extraction of all spectra. Here the bold circle indicates the source spectral extraction region used, of radius of $40^{\prime \prime}$. The dashed areas indicate the background regions used in the extractions; (i) a box along the edge of the chip (but excluding the source region) or (ii) an offset circle of radius $80^{\prime \prime}$.

inclusion of the noisy pixels in the background spectrum could effect the detection of soft X-ray lines. However in order to produce a clean image and event list for GRB 011211, the dataset was screened to include only good X-ray events (using the selection expression "FLAG $=0$ ", in the SAS tool evselect) using only the well-calibrated single or double pixel events (i.e. PATTERN < = 4). An energy cut was also applied, excluding all data below $200 \mathrm{eV}$ and above $10 \mathrm{keV}$. The resulting image is shown in Fig. 2b, which shows a smooth distribution of background counts, regardless of position on the EPIC-pn chips.

Source spectra for GRB 011211 were subsequently extracted from a circular region of $40^{\prime \prime}$ radius, centred on the source. The decrease in flux incurred through photons falling onto the chip gaps was taken into account in the corrected spectra. A background spectrum was taken from a circular region of radius $80^{\prime \prime}$, using a source-free region located close

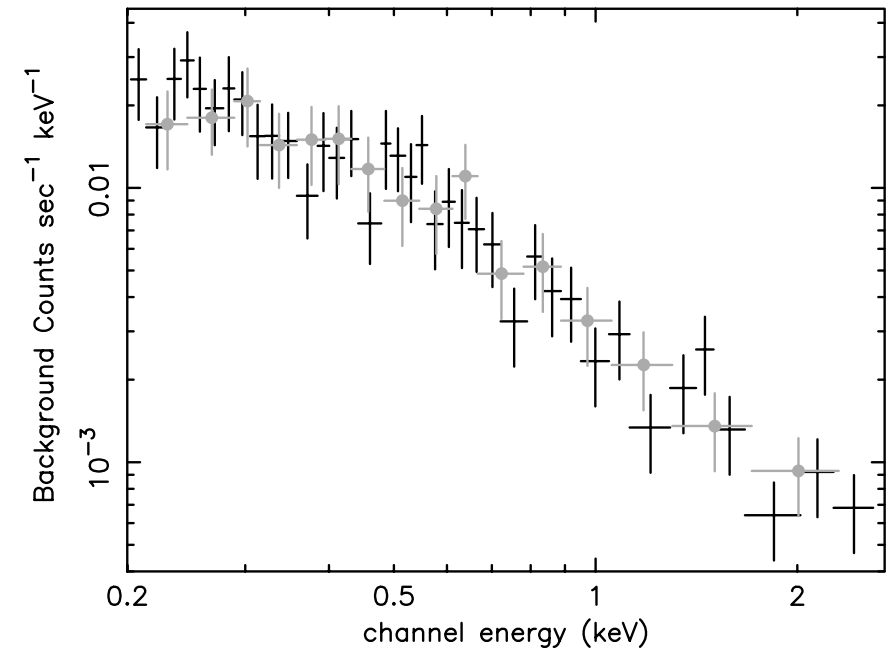

Fig. 3. Background spectra used for spectral analysis during the initial $5 \mathrm{ks}$, the grey circles show the spectrum extracted from the offset circular background region, whilst the crosses show the background spectrum extracted from the box region, located close to the chip edge. The two background spectra are identical, and demonstrate that the background spectrum obtained is both featureless and independent of the position on the pn chip. Overall the background count rate is $\sim 12 \%$ of the net count rate from GRB 011211. Note that the background spectra have been scaled to the size of the source extraction region.

to the source (Fig. 2b). The background spectrum obtained is shown in Fig. 3; after applying the above screening the background spectrum is smooth, unlike the background spectrum obtained by Borozdin \& Trudolyubov (2003), who using unscreened data obtain a "spike" in their background spectrum near $0.7 \mathrm{keV}$. However, in order to ascertain whether the choice of background region can affect the spectrum of GRB 011211, we also use a background extracted from a box-shaped area located close to the EPIC-pn chip gap (Fig. 2b), but excluding the source region. The background spectra obtained for both regions are identical (Fig. 3), hence the choice of background region does not effect the background subtracted source spectrum of GRB 011211. When normalized to the size of the source extraction region, the background contributes $\sim 12 \%$ of the net source count rate for the EPIC-pn.

\section{Spectral analysis}

The background subtracted EPIC-pn source spectrum of GRB 011211, from the first $5 \mathrm{ks}$ of the observation, was fitted in XSPEC V11.1, using a minimum of 12 source counts per bin. The net count rates obtained during the first $5 \mathrm{ks}$ of observation are 0.12 counts $\mathrm{s}^{-1}$ for the pn and 0.018 counts $\mathrm{s}^{-1}$ averaged over the MOS 1 and MOS 2 detectors. An absorbing column of $4.2 \times 10^{20} \mathrm{~cm}^{-2}$, due to our own galaxy, was included in all the spectral fits. A simple power-law fit (model 1) gives a barely (10\% null hypothesis probability) acceptable fit to the data $\left(\chi^{2} /\right.$ d.o.f. $=54.2 / 45$, where d.o.f. is the number of degree of freedom), with a power-law photon index of $\Gamma=2.18 \pm 0.10$. The model EPIC-pn fits to GRB 011211 are summarized in Table 1. Note all the fits are performed with the EPIC-pn detector only, unless otherwise stated. 


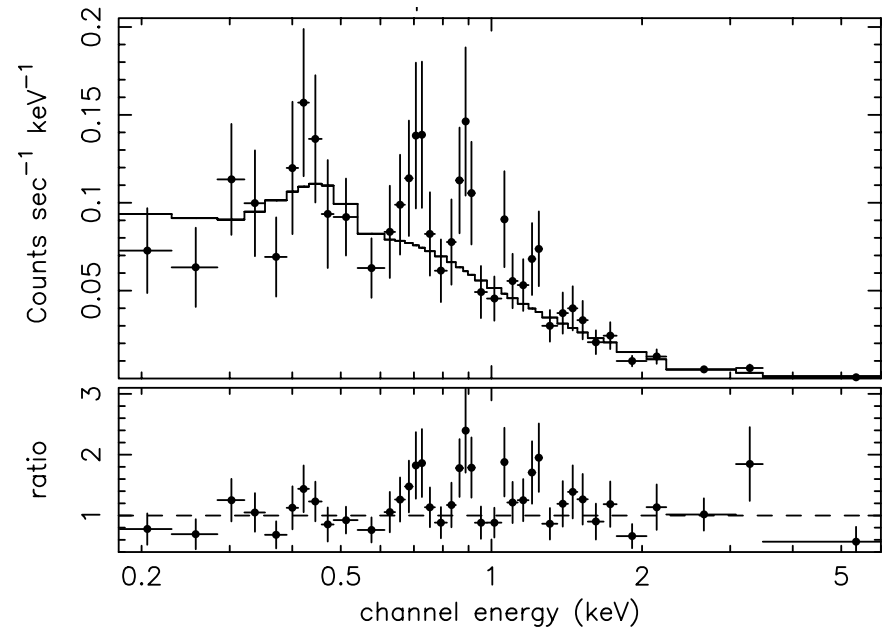

Fig. 4. The background subtracted XMM-Newton EPIC-pn spectrum of GRB 011211, from the first $5 \mathrm{ks}$ of observation. Energy (units keV), in the observed frame, is plotted on the abscissa. In the upper panel, the EPIC-pn data points are plotted with filled circles, whilst the solid line shows a power-law model, folded through the EPIC-pn response function. The lower panel shows the ratio of the data to the power-law fit; an excess of counts is observed between 0.5 and $1.5 \mathrm{keV}$ in the spectrum. This excess can be well fitted with at least 3 emission lines, at $0.71 \pm 0.02 \mathrm{keV}, 0.88 \pm 0.02 \mathrm{keV}$ and $1.22 \pm 0.03 \mathrm{keV}$ respectively.

\subsection{Soft X-ray emission line fits to GRB 011211}

Inspection of the power-law fit to the spectrum of GRB 011211 shows a clear excess present in the data residuals between 0.5 and $1.5 \mathrm{keV}$ (Fig. 4). The deviations are considerably larger than the current calibration uncertainty of the EPIC-pn detector, which is thought to be $<5 \%$ (also see Sect. 3.5); adding a further systematic error of $5 \%$ to the data has no noticeable effect on the spectral fits. Hence, following the procedure of Reeves et al. (2002), we attempt to fit this excess with a set of emission lines. Initially 3 narrow spectral lines were added (model 2), allowing the energy and flux of each line to vary, whilst the line widths were kept negligibly small (at $\sigma=10 \mathrm{eV}$ ) in the fits, as the lines are unresolved compared to the detector resolution. The best fit (observed frame) energies of the lines were then $0.71 \pm 0.02 \mathrm{keV}, 0.88 \pm 0.02 \mathrm{keV}$ and $1.22 \pm 0.03 \mathrm{keV}$, with line fluxes of $(9.9 \pm 3.4) \times 10^{-6}$ photons $\mathrm{cm}^{-2} \mathrm{~s}^{-1},(7.7 \pm 2.3) \times 10^{-6}$ photons cm $\mathrm{cm}^{-2} \mathrm{~s}^{-1}$ and $(4.0 \pm$ 1.4) $\times 10^{-6}$ photons $\mathrm{cm}^{-2} \mathrm{~s}^{-1}$ respectively. The fit is significantly improved, at $99.7 \%$ confidence, upon the addition of these lines $\left(\chi^{2} /\right.$ d.o.f. $=33.7 / 39, \Delta \chi^{2}=20.5$ for 6 extra degrees of freedom), when an F-test is employed (Bevington \& Robinson 1992). Note that even when fitted individually, the lines are detected at 98\%, 97\% and 93\% confidence (for Si, S and Ar respectively), with the addition of 2 extra degrees of freedom (energy, flux) for each line.

Examination of the data/model residuals in Fig. 4 suggests that two further lines can be added to the spectrum (model 3), with observed energies $0.44 \pm 0.04 \mathrm{keV}, 1.46 \pm 0.07 \mathrm{keV}$ and fluxes $(1.0 \pm 0.8) \times 10^{-5}$ photons $\mathrm{cm}^{-2} \mathrm{~s}^{-1}$ and $(1.9 \pm 1.1) \times$ $10^{-6}$ photons $\mathrm{cm}^{-2} \mathrm{~s}^{-1}$ respectively, although the improvement in fit statistic is marginal (only $1 \sigma$ confidence) for these last two cases $\left(\chi^{2} /\right.$ d.o.f. $\left.=29.0 / 35\right)$. Hence a definite detection of

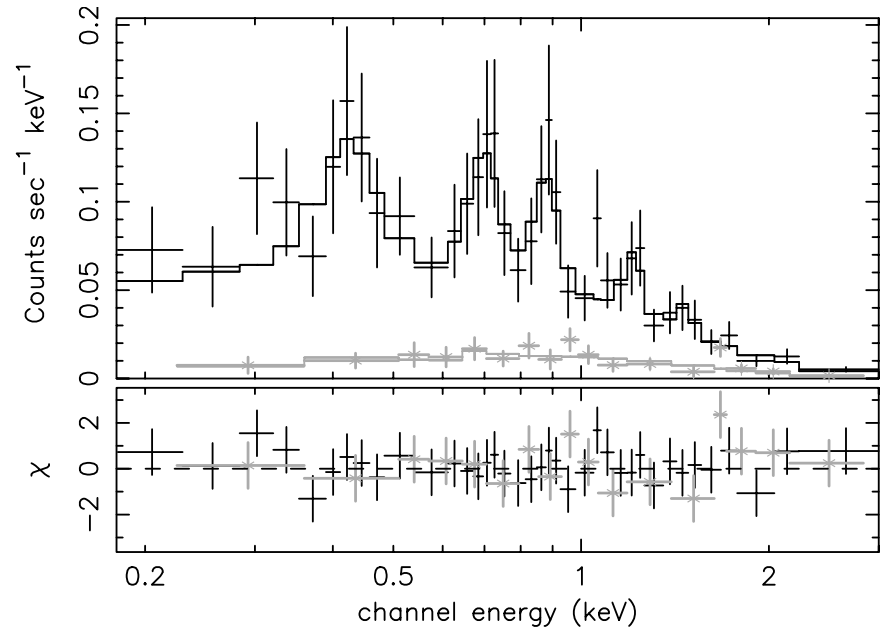

Fig. 5. The XMM-Newton spectrum of GRB 011211, fitted with the redshifted emission line model. The EPIC-pn spectrum is shown in black whilst the lower signal to noise EPIC-MOS spectrum is shown in grey. The lines fitted to the data correspond to Mg XI (or Mg XII), Si XIV, S XVI, ArXVIII and CaXX at a redshift of $z=1.91 \pm 0.06$, implying an outflow velocity of $\sim 0.1 c$ for the emitting material.

Table 1. Summary of X-ray EPIC-pn spectral fits to the first $5 \mathrm{ks}$ of observation for GRB 011211. ${ }^{a}$ Fit statistic, in terms of $\chi^{2}$ divided by degrees of freedom. ${ }^{b}$ Significance of fit improvement, compared to a power-law, from using an F-test. ${ }^{f}$ indicates parameter is fixed in fit.

\begin{tabular}{lccc}
\hline \hline Model & $\Gamma$ or $k T$ & $\chi^{2} /$ d.o.f $^{a}$ & Prob $^{b}$ \\
\hline 1. PL Only & $2.18 \pm 0.10$ & $54.2 / 45$ & \\
2. PL + 3 lines & $2.15 \pm 0.11$ & $33.7 / 39$ & $99.7 \%$ \\
3. PL + 5 lines & $2.10 \pm 0.15$ & $29.0 / 35$ & $99.3 \%$ \\
4. PL + z-lines & $2.12 \pm 0.12$ & $34.3 / 39$ & $99.5 \%$ \\
5. PL + VMEKAL & $\Gamma=2^{f}$ & $38.1 / 41$ & $99.8 \%$ \\
& $k T=4.1 \pm 1.6$ & & \\
\hline
\end{tabular}

these two lines cannot be claimed. Table 2 lists the properties of each of the individual lines added to the X-ray spectra.

Figure 5 shows the EPIC-pn and co-added EPIC-MOS data fitted with the 5 emission lines. Unfortunately none of the lines are significantly detected in the MOS CCDs, due to the lower count rate, although the data for both detectors are consistent (e.g. the residuals in the lower panel are to the same fit to both detectors). If one tries to fit the MOS data alone, during the first $5 \mathrm{ks}$, it is still possible to obtain upper limits to the strongest emission lines at $0.71 \mathrm{keV}$ and $0.88 \mathrm{keV}$ (the putative $\mathrm{Si}$ and $\mathrm{S}$ lines). The values obtained are $<1.4 \times 10^{-5}$ photons $\mathrm{cm}^{-2} \mathrm{~s}^{-1}$ and $<1.1 \times 10^{-5}$ photons $\mathrm{cm}^{-2} \mathrm{~s}^{-1}$ respectively, fully consistent with the measurements made by the EPIC-pn detector.

When converted to the rest frame of the burst (at $z=2.14$ ), the energies of all 5 possible lines are $1.38 \pm 0.10 \mathrm{keV}, 2.22 \pm$ $0.06 \mathrm{keV}, 2.78 \pm 0.05 \mathrm{keV}, 3.83 \pm 0.08 \mathrm{keV}$ and $4.6 \pm 0.2 \mathrm{keV}$. Interestingly these lines all appear to be offset from the known energies of the strong K-shell lines of MgXI $(1.35 \mathrm{keV})$, Si XIV $(2.01 \mathrm{keV})$, S XVI $(2.62 \mathrm{keV})$, Ar XVIII $(3.32 \mathrm{keV})$ and CaXX $(4.10 \mathrm{keV})$. With the exception of Mg XI (the most tentative detection), these lines appear to correspond to the Lyman- $\alpha$ transitions of the most abundant elements, and are 
Table 2. Soft X-ray EPIC-pn line fits to GRB 011211. ${ }^{a}$ Known (laboratory) energy of emission line (in keV); ${ }^{b}$ observed energy (in keV) of emission line in spectrum; ${ }^{c}$ measured rest-frame (at $\left.z=2.14\right)$ energy $(\mathrm{keV})$ of the emission line; ${ }^{d}$ redshift for the emission line, calculated from ${ }^{a}$ and ${ }^{b} .{ }^{c}$ Observed line flux, in units $10^{-6}$ photons $\mathrm{cm}^{-2} \mathrm{~s}^{-1}$.

\begin{tabular}{lccccc}
\hline \hline Ident & $E_{\text {line }}{ }^{a}$ & $E_{\text {obs }}{ }^{b}$ & $E_{\text {rest }}{ }^{c}$ & redshift $^{d}$ & Flux $^{e}$ \\
\hline Mg XI & 1.35 & $0.44 \pm 0.04$ & $1.38 \pm 0.10$ & $2.07 \pm 0.22$ & $10 \pm 8$ \\
Si XIV & 2.01 & $0.71 \pm 0.02$ & $2.22 \pm 0.06$ & $1.84 \pm 0.08$ & $9.9 \pm 3.4$ \\
S XVI & 2.62 & $0.88 \pm 0.02$ & $2.78 \pm 0.05$ & $1.97 \pm 0.06$ & $7.7 \pm 2.3$ \\
Ar XVIII & 3.32 & $1.22 \pm 0.03$ & $3.83 \pm 0.08$ & $1.72 \pm 0.07$ & $4.0 \pm 1.4$ \\
Ca XX & 4.07 & $1.46 \pm 0.07$ & $4.6 \pm 0.2$ & $1.80 \pm 0.13$ & $1.9 \pm 1.1$ \\
\hline
\end{tabular}

the strongest emission lines one would expect to observe at these X-ray energies for either a collisionally ionised plasma (of temperature a few keV) or a highly photoionised plasma.

Having established the likely identity of the soft X-ray line features in GRB 011211, we then calculated the observed redshift, and thus the outflow velocity of the line emitting material. Fitting the redshift of the lines individually, one finds values of $z=2.07 \pm 0.22(\mathrm{Mg} \mathrm{XI}), z=1.84 \pm 0.08$ (Si XIV), $z=1.97 \pm 0.06(\mathrm{~S} \mathrm{XVI}), 1.72 \pm 0.07$ (Ar XVIII) and $1.80 \pm 0.13$ (CaXX). Thus with the exception of Mg XI (which at the detector resolution is unresolved from $\mathrm{Mg}$ XII and also L-shell emission from $\mathrm{Fe}$ or $\mathrm{Ni}$ ) the lines all appear to be significantly offset from the known redshift for GRB 011211 of $z=2.140 \pm$ 0.001 , corresponding to an outflow velocity of $\sim 0.1 c$. In order to test the significance of this, we fixed the line energies at these known rest-frame values, but allowed a single redshift (between $z=0$ and $z=10$, in steps $\Delta z=0.05$ ) for the entire line set to vary (model 4$)$. The best-fit redshift calculated for the line set is $z=1.91 \pm 0.06$, corresponding to an outflow velocity of $0.09 \pm 0.02 c$ or $\sim 25000 \mathrm{~km} \mathrm{~s}^{-1}$ from the GRB progenitor. Overall, the fit statistic improves to $\chi^{2} /$ d.o.f. $=34.3 / 39$. When compared to the power-law fit (for 6 extra degrees of freedom), the addition of the redshifted line set is significant at $99.5 \%$ confidence from an F-test.

Calculating a weighted mean of the 5 line redshifts gave a mean of $z=1.86 \pm 0.07$, consistent with the best-fit value derived above. However, to see whether there are any significant variations in redshift for any individual lines, we calculated the total $\chi^{2}$ deviations from the weighted mean redshift (of $z=1.86 \pm 0.07$ ) for each of 5 lines. This gave a total $\chi^{2}$ value of 8.5 for 4 degrees of freedom, with a corresponding null hypothesis probability of $7 \%$. Alternatively if one uses the best-fit redshift of $z=1.91 \pm 0.06$ from above, then the $\chi^{2}$ is 10.4 for 4 degrees of freedom; the corresponding null hypothesis probability is $3 \%$.

Whilst these results may be deemed as being (barely) acceptable and consistent with a constant redshift for each line, we cannot preclude the possibility of some intrinsic deviation in redshift for each individual element. The Ar XVIII line shows the biggest discrepency from the best-fit value of $z=1.91 \pm 0.06$ (at $2.6 \sigma$ ), with a redshift of $1.72 \pm 0.07$. However it is also possible that the $1.22 \mathrm{keV}$ feature could instead be identified with the radiative recombination continuum of $\mathrm{S} X \mathrm{XVI}$ at $3.49 \mathrm{keV}$, which would instead imply a redshift of $z=1.86$, consistent with the mean value. The would imply that the plasma is strongly recombining, either due to heavy cooling (which would be achieved at high densities) or photoionisation (e.g. see Piro et al. 2000 for the possible detection of iron re-combination emission). Alternatively, any variations in redshift may just imply that the different elements are ejected with a range of velocities from the burst progenitor. Future observations at higher signal to noise and at higher spectral resolution are required to test this possibility.

\subsection{Thermal emission and ionised reflection models}

Finally, we attempted to fit the data with emission from a collisionally ionised optically thin plasma, with variable elemental abundances (hereafter the "thermal" emission model), using the VMEKAL code within XSPEC (Mewe et al. 1985). Initially, a thermal emission component of temperature $k T=$ $4.1 \pm 1.6 \mathrm{keV}$ was added in addition to an underlying powerlaw component (model 5). The improvement in fit obtained $\left(\Delta \chi^{2} /\right.$ d.o.f. $\left.=38.1 / 41\right)$ is significant when compared to the pure power-law fit (model 1); the thermal emission component is required at $99.8 \%$ confidence from an F-test, for four extra degrees of freedom (temperature, abundance, redshift and luminosity). Note that the spectrum during the initial $5 \mathrm{ks}$ can be equally well fitted with pure thermal emission (i.e. the powerlaw component is not statistically required) the fit statistic is then $\chi^{2} /$ d.o.f. $=38.5 / 43$. The rest-frame model spectrum using the VMEKAL component is shown in Fig. 6. It can be seen that for these elements, the hydrogenic states dominate (e.g. Si XIV at $2.0 \mathrm{keV}$ ), although there is also some contribution from the He-like species as well (e.g. S XV, Ar XVII, Ca XIX).

We also used this model to provide an independent determination of the redshift of the line emitting material, where no assumption has been made about the likely identity of the lines; the best-fit redshift was $1.89 \pm 0.08$, in excellent agreement with the simple line fit derived above. A contour plot showing the $68 \%, 90 \%, 95 \%, 99 \%$ and $99.9 \%$ confidence levels for the line emitting material is shown in Fig. 7, a redshift of $z=2.14$ can be ruled out at $>99 \%$ confidence. Rutledge \& Sako (2003) suggest that emission at $z=1.2$ and $z=2.75$ are also plausible solutions to the data, clearly these are ruled out from our fits, although there is a local minimum at $z \sim 1.2$ (which can be excluded at $\sim 99 \%$ confidence). Nonetheless, we consider both the lower redshift of $z=1.2$ and the high redshift value of $z=2.75$ to be physically implausible, as this would require either an extreme blueshift or for the GRB to reside at a higher 


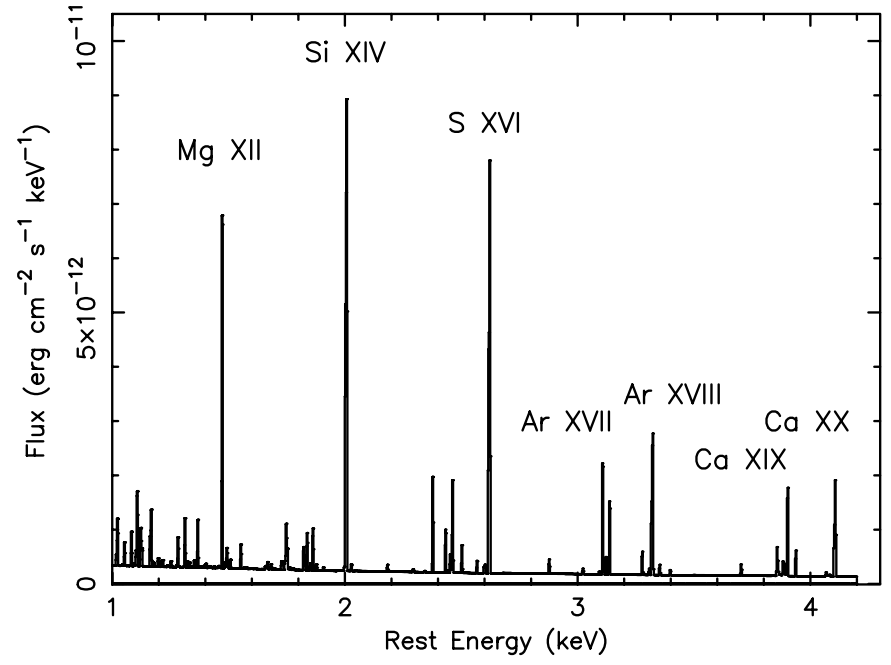

Fig. 6. Rest-frame thermal emission model showing the relative strengths of the major emission lines (and continuum) for a plasma of temperature $4 \mathrm{keV}$.

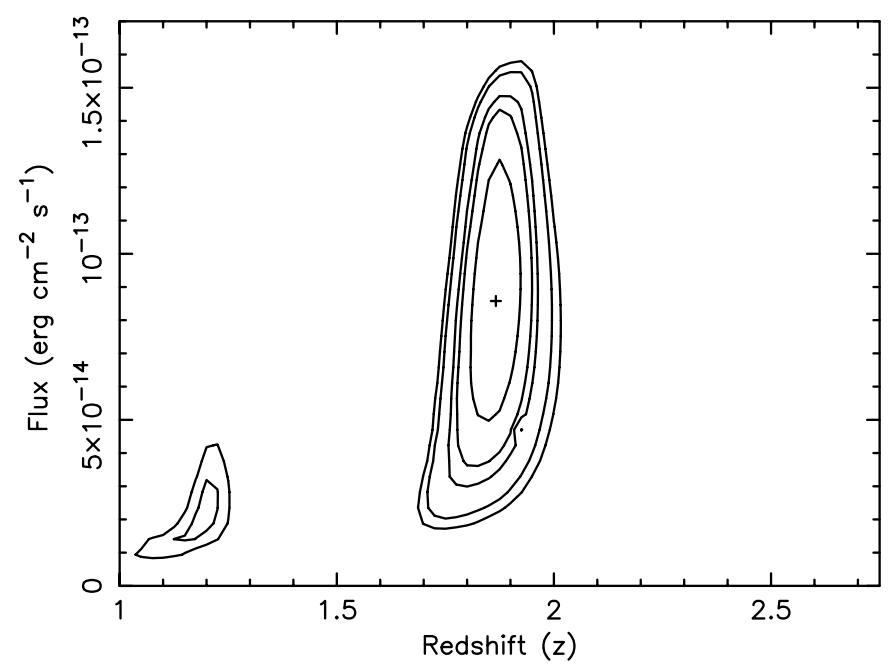

Fig. 7. Confidence contour plot of measured flux against redshift for the thermal line emission model, added in addition to a power-law component. The contours represent the $68 \%, 90 \%, 95 \%, 99 \%$ and $99.9 \%$ confidence levels to the spectral fit, whilst the redshift has been varied (in $\Delta z=0.05$ increments) between $z=1$ and $z=3$. The thermal emission is required in the data at $>99.9 \%$ confidence, whilst the need for an outflow velocity, with respect to the GRB rest frame, is also required at $>99 \%$ confidence.

redshift than the measured value (from the optical spectrum) of $z=2.14$.

From a pure thermal plasma fit, the relative abundance of the light metals (Mg, Si, S, Ar, Ca) was found to be $\sim 9 \times$ the solar value, with a $90 \%$ lower-limit of $4 \times$ solar (and note that the inclusion of an addition power-law component in the fits has the effect of increasing the required abundance for the light metals). Interestingly, unlike the other elements, an over abundance of iron is not required, the $90 \%$ upper limit for the iron abundance was found to be $<1.4 \times$ solar, consistent with the lack of detection $(E W<400 \mathrm{eV}$, rest frame) of a strong iron $\mathrm{K}$-shell line in the data.

We also attempted to fit the spectrum with a reflection model (Ballantyne \& Ramirez-Ruiz 2001), where the X-rays scatter off the walls of a funnel excavated in the envelope of a stellar progenitor (the so-called "nearby reprocessor" model, Rees \& Meszaros 2000). Firstly, we tried a pure reflection component (i.e. just the scattered X-ray emission). Allowing the ionisation state of the reflecting material to vary results in an ionisation parameter $\log \xi>4$ (with $\Gamma=2.0$ ) and a fit statistic of $\chi^{2} /$ d.o.f. $=55.3 / 44$. In this fit, the spectrum resembles a simple power-law, as all the elements are fully ionised, and the reflector is a perfect mirror. If one forces the ionisation parameter to be $\log \xi<4$, then the fit statistic is worse $\left(\chi^{2} /\right.$ d.o.f. $\left.=66.7 / 43\right)$, whilst the incident power-law becomes steep $(\Gamma \sim 3)$. The poor-fit results from the strong iron line or edge present in the model, and from the shape of the continuum, which is concave in the reflection model (but is convex in the actual data). Allowing a direct power-law component in addition to the reflector does not improve the fit further. Hence it is difficult to reconcile the current reflection models with the spectrum of GRB 011211, although we note that at present, this model does not include emission from $\mathrm{S}$, Ar or Ca.

\subsection{Monte-Carlo simulations}

We have used Monte-Carlo techniques to provide an independent estimate of the significance of the line emission fits to GRB 011211. This is important given the low photon number statistics of the data, where the distribution of counts may become non-Gaussian (Protassov et al. 2002; Yaqoob 1998).

In order to test the significance of the line emission, 10000 random spectra from the EPIC-pn detector were generated, using a simple power-law emission model (including absorption from our own Galaxy), with a photon index of $\Gamma=2.2$. An exposure time of $5 \mathrm{ks}$ (accounting for detector deadtime) and an X-ray flux identical to that of GRB 011211 during the first $5 \mathrm{ks}$ of observation $\left(F_{0.2-10 \mathrm{keV}}=2.1 \times\right.$ $\left.10^{-13} \mathrm{erg} \mathrm{cm}^{-2} \mathrm{~s}^{-1}\right)$ were used, as well as a background level $\sim 10 \%$ of the source count rate, so that the signal to noise of the simulated spectra were comparable to the actual source spectrum for GRB 011211. The simulated spectra were rebinned to obtain a minimum of 12 source counts in each spectral channel.

We proceeded to fit the simulated data, using (i) a pure power-law continuum and (ii) a model consisting of a powerlaw plus three emission lines, corresponding to the strongest lines ( $\mathrm{Si}, \mathrm{S}, \mathrm{Ar}$ ) observed in the actual GRB 011211 spectrum. The difference in $\Delta \chi^{2}$ between the power-law and line emission models was noted for each simulated spectrum, in order to test whether the apparent line emission could be produced purely through random, Poisson noise. Intially, the line energies were fixed at the rest-frame values corresponding to the $\mathrm{K} \alpha$ transitions of Si XIV, S XVI and Ar XVIII, but note that the redshift was allowed to vary between $z=0$ and $z=10$ (stepsize $\Delta z=0.05$ ) when fitting the trial spectra. Hence no prior assumption is made about the redshift of the line emitting material. In addition, the line flux for each line was allowed 
to vary in the fits. Upon fitting the simulations we found that only 5 in 10000 of the randomly generated power-law spectra could yield a similar decrease in chi-squared $\left(\Delta \chi^{2} \sim 20\right)$, when compared to the real data, after the addition of the three emission lines. The likelihood (or null hypothesis probability) that the lines could arise purely from Poisson noise in this test is $0.05 \%$; hence the simulations indicate that the soft X-ray line emission is detected at $99.95 \%$ confidence, in agreement with the results obtained through the F-test.

In a recent paper, Rutledge \& Sako (2003) note that the prior identification of the three strongest emission line features with Si XIV, S XVI and ArXVIII represents an a posteriori assumption about the data, when trying to estimate the significance of the line features. However we note (and see Fig. 6) that line emission features from these 3 abundant $\mathrm{H}$-like species will dominate the emission from a hot (several $\mathrm{keV}$ ) collisionally ionised plasma, or a highly photoionised plasma, over the 1-4 keV (rest frame) energy range. Nonetheless we relax the assumption that the lines originate from these species, and test the improvement in fit obtained when adding lines, at any energy from $0.2-10 \mathrm{keV}$ in the observed spectrum, to the 10000 fake power-law spectra (i.e. a blind spectral search). Initially we just add one line to the fake spectra (whilst varying both the energy and flux of the line), and find that an improvement of $\Delta \chi^{2}=8$, equivalent to the addition of the Si XIV line in the real dataset, is obtained in only 6 out of 1000 cases. Thus the Si XIV line is detected at $99.4 \%$ confidence. Similarly we find that the S XVI and Ar XVIII lines are detected at $98.8 \%$ and $96 \%$ confidence respectively, in agreement with the values computed by the F-test. We also consider the significance of the 3 line set as a whole, by fitting 3 Gaussian lines (again at arbitrary energies) to the simulated datasets. The results (see Fig. 8a) show that only 3 in 10000 trial spectra gave an equivalent (or better) improvement in the $\chi^{2}$ statistic compared to the real data (where $\Delta \chi^{2}=20.5$ for the addition of all 3 lines). Thus we can conclude that the addition of 3 Gaussian lines in the soft X-ray spectrum of GRB 011211 is significant at $>99.9 \%$ confidence, whilst the individual lines are detected at $99.4 \%, 98.6 \%$ and $96 \%$ confidence (for $\mathrm{Si}, \mathrm{S}$ and $\mathrm{Ar}$ ) respectively.

A more physically realistic model, consisting of emission from a hot, thermal plasma (i.e. the VMEKAL model), was also fitted to the 10000 randomly generated power-law spectra. The temperature, luminosity, redshift and relative abundance of the plasma were allowed to vary in the fits. Figure 8 (panel b) shows a histogram of the change in $\chi^{2}$ obtained when comparing a power-law fit to a thermal fit for the simulated spectra; note here a positive change in $\chi^{2}$ corresponds to an improvement in fit statistic when comparing the VMEKAL model to a power-law. In the majority (90\%) of cases, fitting a thermal model to the randomly generated power-law spectra results in a worse fit. However the real data for GRB 011211 indicate that an improvement in fit statistic of $\Delta \chi^{2}=15.7$ is obtained using the thermal model compared to the pure power-law. Indeed an equivalent improvement in $\Delta \chi^{2}$ is only found in 3 out of 10000 simulated spectra; hence the inclusion of the thermal line emission component is formally required at $>99.9 \%$ confidence from the simulations.
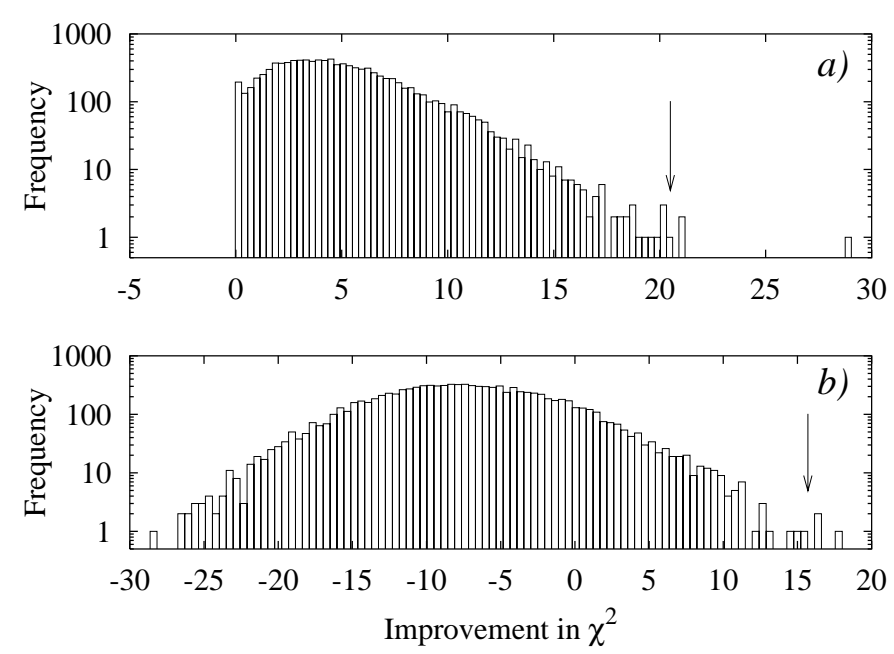

Fig. 8. Histograms showing the improvements in the fit statistic $\left(\chi^{2}\right)$ obtained when fitting 10000 simulated power-law spectra with; a) a model consisting of a power-law plus three emission lines (with the line energies and fluxes both allowed to vary) and b) the thermal (VMEKAL) emission model. In both cases, only three out of 10000 randomly generated trial spectra yield an improvement in $\chi^{2}$ equivalent to that found in the actual observation; note the $\Delta \chi^{2}$ for the real GRB 011211 data is marked by an arrow. The simulations show that the probability of generating the soft X-ray line emission through random Poisson noise is only $0.03 \%$.

\section{Spectral binning, extraction and calibration}

\subsection{The effect of spectral binning}

The effect of spectral binning was also investigated. Rather than binning the spectrum to a set number of photons per bin, the spectra were re-binned so that each bin represented a constant energy interval, of $40 \mathrm{eV}$ in size. This is then well matched to the resolution of EPIC-pn detector below $2 \mathrm{keV}$ (where the FWHM spectral resolution is $\sim 80 \mathrm{eV}$ ). Data above $2 \mathrm{keV}$ were ignored due to poor photon statistics (less than 10 source counts per bin). The resultant spectrum is shown in Fig. 9. An initial power-law fit with Galactic absorption yields a poor fit with $\chi^{2} /$ d.o.f. $=60.9 / 42$. As in Sect. 3.1, the spectrum has been fitted with the 5 redshifted lines obtained previously, the fit statistic again improves significantly (from $\chi^{2} /$ d.o.f. $=$ $60.9 / 42$ to $33.4 / 32$, i.e. at $99 \%$ confidence) upon the addition of the line set. Interestingly, a residual near $1.1 \mathrm{keV}$ is present in the data, the addition of a sixth emission line, at $1.09 \pm 0.02 \mathrm{keV}$, is significant at $98 \%$ confidence $\left(\chi^{2} /\right.$ d.o.f. $=$ 25.9/30). Assuming a redshift of $z=1.9$, this line could correspond to He-like $\operatorname{Ar}$ (Ar XVII), which is predicted in the thermal model (Fig. 5).

The net redshift obtained for the line set is then $z=1.86 \pm$ 0.04 , consistent with the previous determination (Sect. 3.1). The observed-frame energies for the $5 \mathrm{H}$-like lines are measured to be $0.43 \pm 0.04 \mathrm{keV}, 0.70 \pm 0.02 \mathrm{keV} 0.89 \pm 0.02 \mathrm{keV}$, $1.23 \pm 0.03 \mathrm{keV}, 1.45 \pm 0.05 \mathrm{keV}$, whilst the observed line fluxes are then $1.8 \pm 1.2 \times 10^{-5}$ photons $\mathrm{cm}^{-2} \mathrm{~s}^{-1}, 1.3 \pm 0.5 \times 10^{-5}$ photons $\mathrm{cm}^{-2} \mathrm{~s}^{-1}, 9.2 \pm 3.5 \times 10^{-6}$ photons $\mathrm{cm}^{-2} \mathrm{~s}^{-1}, 4.2 \pm 2.2 \times$ $10^{-6}$ photons $\mathrm{cm}^{-2} \mathrm{~s}^{-1}$ and $2.4 \pm 1.5 \times 10^{-6}$ photons $\mathrm{cm}^{-2} \mathrm{~s}^{-1}$ respectively. Within the errors, the measured energies of fluxes 


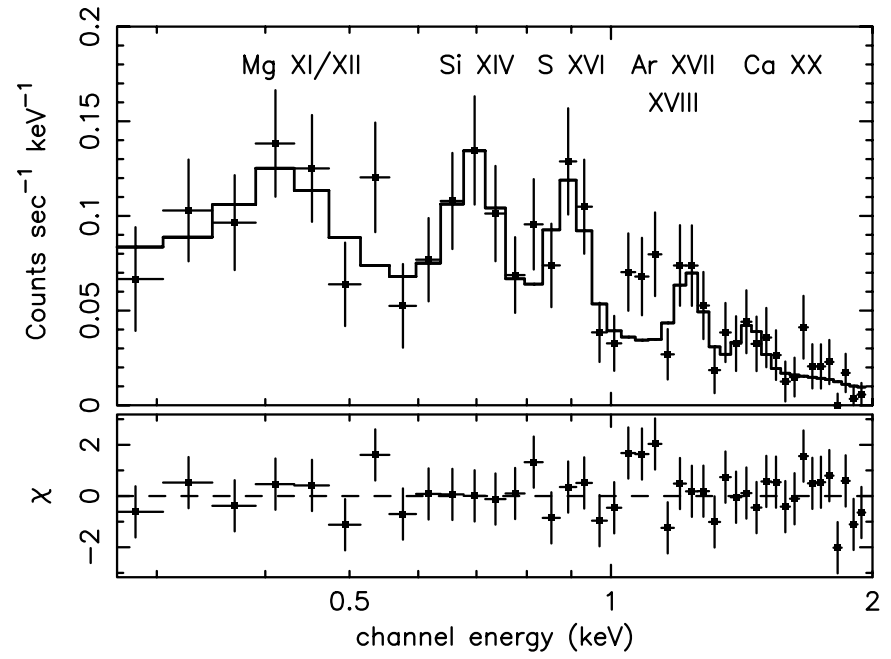

Fig. 9. EPIC-pn spectrum of GRB 011211, binned into equal energy bins, $40 \mathrm{eV}$ in size. The 5 redshift emission lines are clearly detected, and a sixth line at $1.1 \mathrm{keV}$, due to He-like Ar (Ar XVII) may also be present (at 98\% confidence). The likely identifications of the lines are marked at the top of the plot.

agree remarkably well with those in Sect. 3.1 (see Table 2), which indicates that the energy binning scheme used has little effect on the derived spectral fits to the data. The thermal emission line model (see Sect. 3.2), was also fitted to the rebinned data. The best fit parameters obtained were $k T=2.9 \pm 0.6 \mathrm{keV}$ for the plasma temperature, redshift $z=1.86 \pm 0.07$, whilst the relative abundance of the light metals were found to be $5.5 \pm 2.2$ times the solar value. These are consistent with the parameters derived in section 3.2. Similarily the thermal plasma fit (with fit statistic $\chi^{2} /$ d.o.f. $=42.4 / 40$ ) is statistically preferred over the power-law fit (at $>99.9 \%$ ) confidence.

Finally we also used the $C$-statistic (see Cash 1979) to compute the best-fit spectrum for GRB 011211. Interestingly the best-fit value for the $C$-statistic obtained for a power-law fit, plus 5 emission lines (as detailed above) is 33.5 for 44 pha bins, almost identical to the value of $\chi^{2}$ obtained above for the same model fit. The model fit parameters obtained are identical regardless of whether $\chi^{2}$ or $C$ statistics are used.

\subsection{Varying the source extraction radius}

We have investigated whether varying the size of the source selection region has any effect on the derived spectrum and the detection of the soft X-ray lines. In order to test this, we extracted spectra from circular regions of radii $10^{\prime \prime}, 20^{\prime \prime} 30^{\prime \prime}$ in addition to the previous spectrum that was extracted with a $40^{\prime \prime}$ radius. We find the detection of the redshifted soft $\mathrm{X}$-ray line set is significant in each case; at $>99 \%$ confidence (via an F-test) for spectra extracted with $20^{\prime \prime}$ and $30^{\prime \prime}$ radii, whilst the detection is less significant (at $98 \%$ confidence) for a $10^{\prime \prime}$ radius (as substantially fewer source counts are included in the spectrum). However, after accounting for the telescope point spread function, the derived line flux for the Si XIV line was found to be constant in each case (at $\sim 1.0 \times 10^{-5}$ photons $\mathrm{cm}^{-2} \mathrm{~s}^{-1}$ ), in contrast to the findings of Borozdin \& Trudolyubov (2003) who

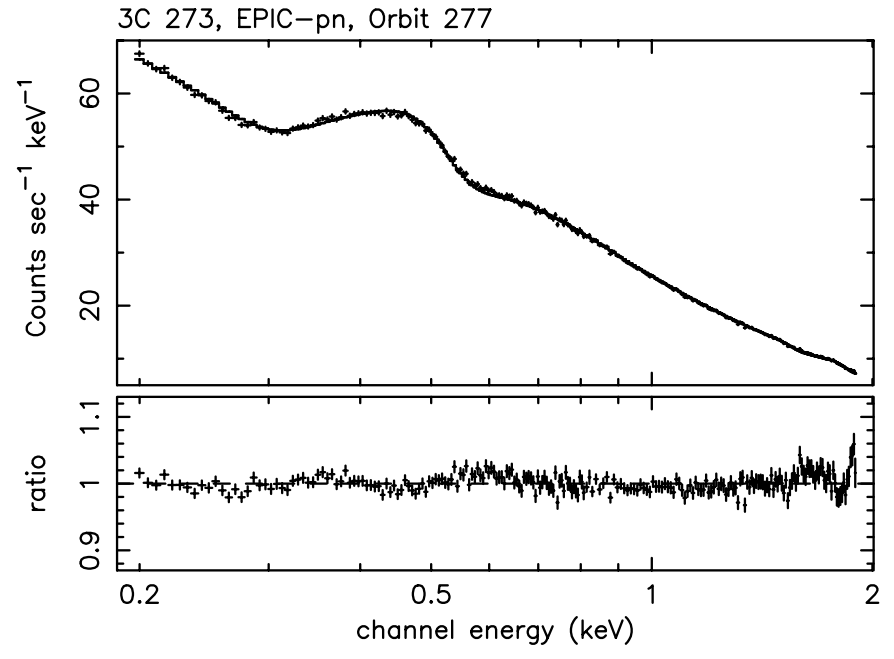

Fig. 10. EPIC-pn count-rate spectrum obtained during the calibration observation of 3C 273 during orbit 277 . The spectrum has been fitted with a simple power-law model, over the energy range $0.2-2.0 \mathrm{keV}$. The lower-panel shows the data/model ratio residuals to this powerlaw fit. No strong deviations are present above a systematic $2 \%$ level, and no strong narrow line-like deviations are seen in the residuals. It is therefore unlikely that the line-like features in the EPIC-pn spectrum of GRB 011211 are caused by artifacts in the pn detector calibration.

claim the Si XIV line flux in their spectra decreases with the extraction radius.

\subsection{Calibration effects of EPIC-pn}

We also investigate whether the calibration of the EPIC-pn detector response could have any effect on the observed emission lines. To do this, we have chosen the publically available calibration observation of the quasar 3C 273 with XMM-Newton, during orbit 277. 3C 273 has been chosen as it is a very bright $\mathrm{X}$-ray source (with a typical X-ray flux of $\sim 10^{-10} \mathrm{erg} \mathrm{cm}^{-2} \mathrm{~s}^{-1}$ ) which is thought to have only weak absorption or emission features in the X-ray band (e.g. Grandi et al. 1997; Reynolds 1997). The EPIC-pn spectrum has been extracted from a circular region of radius $40^{\prime \prime}$ located $40^{\prime \prime}$ away from the edge of the EPIC-pn chip (observed in small window mode). The EPIC-pn data have then been fitted with a single power-law of $\Gamma=2.0$ (plus absorption from our own galaxy of $1.8 \times 10^{20} \mathrm{~cm}^{-2}$ ) over the $0.2-2.0 \mathrm{keV}$ range, where the soft $\mathrm{X}$-ray lines are present in GRB 011211. The count-rate spectrum and data/model residuals are shown in Fig. 10. It can be seen that there are no strong residuals present above a systematic level of $2 \%$ in the spectrum, and no strong narrow emission line features are seen, unlike those in GRB 011211. We conclude that the spectral calibration of the EPIC-pn detector cannot account for the observed spectral features in GRB 011211.

\section{Temporal variability of the line emission in GRB 011211}

The observations presented in Reeves et al. (2002) showed that the line emission in GRB 011211 decayed with time faster than the continuum, rendering the lines undetectable 
after $10 \mathrm{ks}$ of observation. In order to quantify this, we have calculated the ratio of thermal to power-law emission during the first and last $10 \mathrm{ks}$ of observation. During the first $10 \mathrm{ks}$, the thermal component has an average luminosity of $2.6 \pm 1.1 \times 10^{45} \mathrm{erg} \mathrm{s}^{-1}$, whilst the power-law component has a luminosity of $5.7 \pm 1.5 \times 10^{45} \mathrm{erg} \mathrm{s}^{-1}$ (and note that both components are statistically required in the spectral fit at $>99 \%$ confidence). In the last $10 \mathrm{ks}$ the power-law component has a luminosity $5.3 \pm 0.3 \times 10^{45} \mathrm{erg} \mathrm{s}^{-1}$, whilst the thermal emission is no longer required $\left(L<1.07 \times 10^{45} \mathrm{erg} \mathrm{s}^{-1}\right)$.

We have also parameterised the strength of the line emission by measuring the equivalent width $(E W)$ of the Si XIV and S XVI lines relative to a pure (but decaying) power-law continuum. During the first $5 \mathrm{ks}$, the Si XIV line is detected with an (observed frame) $E W$ of $127 \pm 42 \mathrm{eV}$. A line is also marginally detected (at $90 \%$ confidence) from 5-10 ks after the start of the observation, with a smaller $E W$ of $62 \pm 30 \mathrm{eV}$. Beyond $10 \mathrm{ks}$, the line is not detected, we can place a tight upper-limit of $<25 \mathrm{eV}$ on the $E W$ of the line from the last 20 ks of observation. Similarly for the S XVI line, the measured equivalent width during the first $5 \mathrm{ks}$ is $154 \pm 70 \mathrm{eV}$, whilst upper-limits only are obtained from 5-10 ks and during the last $20 \mathrm{ks}$ (the limits are $<30 \mathrm{eV}$ and $<22 \mathrm{eV}$ respectively). Thus the soft X-ray line emission in GRB 011211 appears to decay with time, and none of the lines are detected after $10 \mathrm{ks}$ from the start of the XMM-Newton observation.

\section{Discussion and conclusions}

Both the F-test and Monte-Carlo simulations have showed that the soft X-ray line emission in GRB 011211 is detected at $>99 \%$ confidence, We conclude that the detection of the soft $\mathrm{X}$-ray features in GRB 011211 is robust, and is not affected by the detector background, calibration, spectral binning or the particular spectral model that is assumed. It is also apparent that the lines decay rapidly, no line emission is formally detected after $10 \mathrm{ks}$ from the start of the XMM-Newton observation.

The early afterglow data (during the first $10 \mathrm{ks}$ of observation) appears to be characterised by thermal X-ray emission (from optically thin gas). An alternative spectral model, where the afterglow line emission results from X-ray reflection off optically thick material appears to be ruled out (e.g. Ballantyne \& Ramirez-Ruiz 2001), although we note that in current reflection models, the emission from $\mathrm{S}, \mathrm{Ar}$ and $\mathrm{Ca}$ is not computed. Future models will hopefully provide a better test of the reflection scenario.

On the assumption that the lines result from thermal emission one can estimate the mass of the ejected material. Firstly the emission measure $\left(E_{\mathrm{M}}\right)$ is computed from the observed luminosity of the thermal emission component. Here $n^{2} V=$ $E_{\mathrm{M}} \sim 10^{69} \mathrm{~cm}^{-3}$ (where $n$ is the electron density in $\mathrm{cm}^{-3}$ and $V$ is the volume), whilst the plasma will emit X-rays over a characteristic cooling time (governed by the electron density) of $t_{\text {cool }}=1.4 \times 10^{15} n^{-1} \mathrm{~s}$, for gas of temperature $k T=4 \mathrm{keV}$. However, for the case where the cooling time of the plasma is shorter than the observed lifetime of the lines (i.e. $t_{\text {cool }}<<t_{\text {line }}$ ), which will occur at high plasma densities (see Reeves et al. 2002), then due to time delay effects, the total integrated output of the thermal emission is then governed by $n^{2} V=E_{\mathrm{M}} t_{\text {line }} / t_{\mathrm{cool}}$, where $t_{\text {line }}$ is the observed lifetime of the line emission. Note in this scenario, the lifetime of the emission lines arises through reverberation effects, as $t_{\text {line }}=R / c(1-\cos \theta)$, where $R$ is the distance to the line emitting matter and $\theta$ is the opening angle of the cone or jet.

Substituting for the cooling time, and as $n V=M / m_{\mathrm{p}}$ (where $M$ is the total mass of the emitting material and $m_{\mathrm{p}}$ is the proton mass), then one can derive the total mass of the ejecta:

$$
\begin{aligned}
& M=2.4 \times 10^{30} t_{\text {line }} \mathrm{g} \\
& \text { or } M=1.2 \times 10^{-3} t_{\text {line }} M_{\odot} .
\end{aligned}
$$

The minimum lifetime of the line emission (measured from the start of the XMM-Newton observation) is $10^{4} /(1+z) \mathrm{s}$, whilst the maximum lifetime occurs if the line emission starts at the time of the burst, which is then $t_{\text {line }}=5.5 \times 10^{4} /(1+z) \mathrm{s}$. Thus the minimum and maximum mass of the outflowing material, responsible for the blue-shifted line emission, are then 4 and 20 solar masses respectively. This indicates that the mass of the stellar progenitor is likely to be $>20 M_{\odot}$ even for the conservative case where the mass of the ejecta is $4 M_{\odot}$ (e.g. Woosley \& Weaver 1995).

The duration of the line features in GRB 011211, observed 11 hours after the initial burst, implies a distance of several $\times 10^{15} \mathrm{~cm}$ for the line emitting matter (Reeves et al. 2002). When combined with the velocity of the material $(\sim 0.1 c)$, this implies that the matter was ejected from days to weeks before the burst itself occurred. This time delay appears too short in the context of the "supranova" model (Vietri \& Stella 1999), where a time-delay of several months to years can be predicted, whilst the "hypernova" class of models (Woosley 1993) involve the near-simultaneous occurrence of a supernova and the gamma ray burst. Note however that Lazzati (2003) is able to reconcile the observed thermal emission into a timescale of months to years, consistent with the supranova model, although this does not explain the low abundance of iron. Interestingly one model has been proposed involving the coalescence of a close binary system, resulting from a collapse of a massive stellar progenitor (Davies et al. 2002). This could naturally account for the time delay between the putative supernova and the gamma ray burst, with a delay of a few days resulting from the orbital decay (through gravitational radiation) of the close binary system.

If the line emitting material is located at much smaller distances $\left(R \sim 10^{13} \mathrm{~cm}\right)$, as in nearby reprocessor models (e.g. Rees \& Meszaros 2000) then the need for a significant time delay is reduced. Here, the line emission results from re-processing (via reflection/photoionisation) of continuum photons emitted after the initial burst. However in this scenario, significant continuum emission should be observed at the same time as the line emission, which would render any soft X-ray line features undetectable by current instrumentation (see Lazzati et al. 2002). One possibility that has been suggested (Kumar \& Narayan 2003) is that the ionising photons are scattered back towards the ejected material, by a positronelectron shield generated by the initial $\gamma$-rays themselves. This model removes the need for a supernova-GRB delay, as the 
duration of the line emission is accounted for by the time required for the photons to be scattered back towards the ejected matter.

Including GRB 011211, there have now been 4 detections of GRB afterglows with XMM-Newton (GRB 001025A and GRB 010220, Watson et al. 2002; GRB 020322, Ehle et al. 2002; Watson et al. 2002), whilst the X-ray afterglow for the faint burst GRB 020321 could not be localised. Interestingly, line emission features are also indicated in the X-ray afterglows of GRB 001025A and GRB 010220 (Watson et al. 2002). Similar to GRB 011211, the spectrum of GRB 001025A appears to exhibit a blend of soft X-ray line emission, from the medium- $z$ elements such as $\mathrm{Mg}, \mathrm{Si}, \mathrm{S}$, and Ar. In contrast, the spectrum of GRB 010220 shows a strong iron-group (i.e. $\mathrm{Fe}, \mathrm{Co}$ or $\mathrm{Ni}$ ) line (equivalent width $\sim 1 \mathrm{keV}$ ), although here the $\mathrm{X}$-ray spectrum is absorbed below $1 \mathrm{keV}$, rendering any soft X-ray line emission undetectable. Both fits favour an over abundance of nickel (or cobalt) to iron, which is suggestive of a short time delay (days rather than months) between the putative supernova and burst. Thermal emission models are formally required to fit the soft X-ray excess observed in GRB 001025A, whilst the spectrum of GRB 010220 can be equally well fitted by either thermal or reflection models. Thus soft X-ray line emission is required in at least 2 of the XMM-Newton afterglow spectra (GRB 011211 and GRB 001025A), the exception being the recent burst GRB 020322, which appears to exhibit a featureless (but highly absorbed) power-law spectrum (Watson et al. 2002).

It seems possible that soft $\mathrm{X}$-ray line emission features are relatively common in X-ray afterglow spectra, whilst thermal emission models can fit 3 out of the 4 present XMM-Newton spectra. The previous lack of detection of soft X-ray features with Chandra and Beppo-SAX can be accounted for by the lower effective area of the ACIS-S and LECS detectors respectively in the soft X-ray band. Indeed XMM-Newton is the first $\mathrm{X}$-ray mission with the sensitivity required to detect any putative soft X-ray features in GRBs at energies below $2 \mathrm{keV}$. It is important that XMM-Newton performs follow-up observations of bright $\left(>10^{-13} \mathrm{erg} \mathrm{cm}^{-2} \mathrm{~s}^{-1}\right)$ afterglows, within several hours of the burst, to determine the frequency the soft X-ray line emission. Ultimately, through monitoring the temporal behavior of the line emission from minutes to many hours after a burst, the X-ray telescope on-board NASA's forthcoming Swift mission will be able to trace the distribution of matter around the burst explosion and solve the ambiguities that arise when discriminating between the current GRB models.

Acknowledgements. This paper is based on observations obtained with XMM-Newton, an ESA science mission with instruments and contributions directly funded by ESA Member States and the
USA (NASA). We thank the anonymous referee for his/her comments. James Reeves would like to acknowledge the Leverhulme Trust organisation for their support, in the form of a Research Fellowship. We thank the XMM-Newton SOC for their rapid scheduling of the GRB 011211 observation.

\section{References}

Amati, L., Frontera, F., Vietri, M., et al. 2000, Science, 290, 953

Antonelli, L. A., Piro, L., Vietri, M., et al. 2000, ApJ, 545, L39

Ballantyne, D. R., \& Ramirez-Ruiz, E. 2001, ApJ, 559, L83

Bevington, P. R., \& Robinson, D. K. 1992, Data Reduction and Error Analysis for the Physical Sciences (New York: McGraw-Hill)

Borozdin, K. N., \& Trudolyubov, S. P. 2003, ApJ, 583, L57

Burud, I., Rhoads, J., Fruchter, A., \& Hjorth, J. 2001, GRB Coordinates Network Circular, 1213

Cash, W. 1979, ApJ, 228, 939

Davies, M., King, A., Rosswog, S., \& Wynn, G. 2002, ApJ, 579, L63

Ehle, M., Rodriguez-Pascual, P., Loiseau, N., et al. 2002, GRB Coordinates Network Circular, 1293

Fox, D. W., Bloom, J. S., \& Kulkarni, S. R. 2002, GRB Coordinates Network Circular, 1311

Frontera, F., Amati, L., \& Guidorzi, C. 2001, GRB Coordinates Network Circular, 1215

Grandi, P., Guainazzi, M., Mineo, T., et al. 1997, A\&A, 325, L17

Holland, S. T., Soszynski, I., Gladders, M. D., et al. 2002, AJ, 124, 639

Jansen, F., Lumb, D., Altieri, B., et al. 2001, A\&A, 365, L1

Kumar, P., \& Narayan, R. 2003, ApJ, 584, 895

Lazzati, D. 2003, A\&A, 399, 913

Lazzati, D., Ramirez-Ruiz, E., \& Rees, M. J. 2002, ApJ, 572, L57

Lazzati, D., Ghisellini, G., Amati, L., et al. 2001, ApJ, 556, 471

Mewe, R., Gronenschild, E. H. B. M., \& van den Oord, G. H. J. 1985, A\&AS, 62, 197

Piro, L., Garmire, G., Garcia, M., et al. 2000, Science, 290, 955

Piro, L., Amati, L., Antonelli, L. A., et al. 1998, A\&A, 331, L41

Protassov, R., Van Dyk, D. A., Conners, A., Kashyap, V. L., \& Siemiginowska, A. 2002, ApJ, 571, 545

Rees, M. J., \& Meszaros, P. 2000, ApJ, 545, L73

Reeves, J. N., Watson, D., Osborne, J., et al. 2002, Nature, 416, 512

Reynolds, C. S. 1997, 286, 513

Rutledge, S., \& Sako, M. 2003, MNRAS, in press [astro-ph/0206073]

Santos-Lleo, M., Loiseau, N., Rodriguez, P., Altieri, B., \& Schartel, N. 2001, GRB Coordinates Network Circular 1192

Struder, L., Briel, U., Dennerl, K., et al. 2001, A\&A, 365, L18

Turner, M. J. L., Abbey, A., Arnaud, M., et al. 2001, A\&A, 365, L27

Vietri, L., \& Stella, L. 1999, ApJ, 527, L43

Watson, D., Reeves, J. N., Osborne, J., et al. 2002, A\&A, 393, L1

Watson, D., Reeves, J. N., Osborne, J., et al. 2002b, A\&A, 395, L41

Woosley, S. E., \& Weaver, T. A. 1995, ApJS, 101, 181

Woosley, S. E. 1993, ApJ, 405, 273

Yaqoob, T. 1998, ApJ, 500, 893

Yoshida, A., Namiki, M., Yonetoku, D., et al. 2001, ApJ, 557, L27 